\title{
Bullous Skin Rash: A Rare Case of Palbociclib- Induced Dermatological Toxicity
}

\author{
Noman Ahmed Jang Khan ${ }^{1}$, Mohamed Alsharedi ${ }^{1}$ \\ 1. Hematology and Medical Oncology, Joan C. Edwards School of Medicine at Marshall University, Huntington, USA
}

Corresponding author: Noman Ahmed Jang Khan, noman_jang020@hotmail.com

\begin{abstract}
Palbociclib is an FDA-approved cyclin-dependent kinase inhibitor to treat hormone-positive, HER2-negative metastatic breast cancer. Severe skin toxicities are rare but important adverse events associated with these agents. Early detection of severe forms of skin lesions is crucial to permit the immediate discontinuation of palbociclib in order to avoid unacceptable risk level in the form of severe cutaneous toxicities like StevenJohnson Syndrome. In such cases, palbociclib should be abruptly discontinued and an early aggressive support should be initiated. We here present a case of 50-year-old Caucasian female, who developed acute onset blistering skin lesions one to two weeks after she was started on palbociclib. We sought to report this case given the unusual toxicity and to emphasize the importance of identifying the acute onset of blistering skin lesions, regardless of their extension, should prompt awareness of their potential severity.
\end{abstract}

Categories: Allergy/Immunology, Oncology

Keywords: palbociclib, blistering skin disease

\section{Introduction}

Palbociclib is a well-known cyclin kinase 4/6 inhibitor used in metastatic hormone receptor-positive breast cancer. It is most commonly used as a first-line treatment in conjunction with aromatase inhibitors. Neutropenia, anemia, thrombocytopenia, nausea, diarrhea, and skin rash are some of the reported adverse effects (AEs). The most common skin toxicities are grade 1, ranging from mild urticaria to pityriasis. More severe (grade 3) skin rash was less than $1 \%$ so far in the clinical literature as we found only one case of severe cutaneous toxicity presented as Stevens-Johnson syndrome [1]. Acute bullous skin rash is very rare and warrants immediate discontinuation of palbociclib. We report this case of acute bullous skin rash in a patient who was recently started on palbociclib for metastatic hormone receptor-positive breast cancer.

Received 08/25/2020 Review began 08/29/2020 Review ended 08/30/2020 Published 09/03/2020

() Copyright 2020

Khan et al. This is an open access article distributed under the terms of the Creative Commons Attribution License CC-BY 4.0., which permits unrestricted use, distribution, and reproduction in any medium, provided the original author and source are credited.

\section{Case Presentation}

A 50-year-old female was diagnosed with metastatic hormone receptor-positive breast cancer. She initially presented with hip pain on the right side. MRI of the hip revealed multiple foci of signal abnormalities in the pelvic bones and bilateral femurs. She underwent a right-sided hip replacement, and the bone biopsy revealed a metastatic invasive ductal carcinoma with estrogen receptor (ER) 80\%, progesterone receptor (PR) $15.3 \%$, and human epidermal growth factor 2 (HER2) receptor not overexpressed. Further workup included CT of the chest/abdomen/pelvis and an MRI of the cervical, thoracic, and lumbar spine, revealing extensive bony metastasis throughout the spine and pelvis but no evidence of visceral metastasis. Since the patient was still premenopausal at the time of diagnosis, she was started on tamoxifen along with monthly gosarelin injections for ovarian suppression. After her postmenopausal status was confirmed via hormone levels, she was switched to the aromatase inhibitor anastrazole. After a month, the cyclin-dependent kinase (CDK) 4/6 inhibitor palbociclib was also added to the anastrazole. Two weeks later, she presented to her local oncologist office with a skin rash on her lower extremities. Upon physical examination, fluid-filled bullous lesions were noticed bilaterally on the dorsal surface of her feet with very few ruptured lesions present on the right foot and shin (Figures 1,2). 


\section{Cureus}

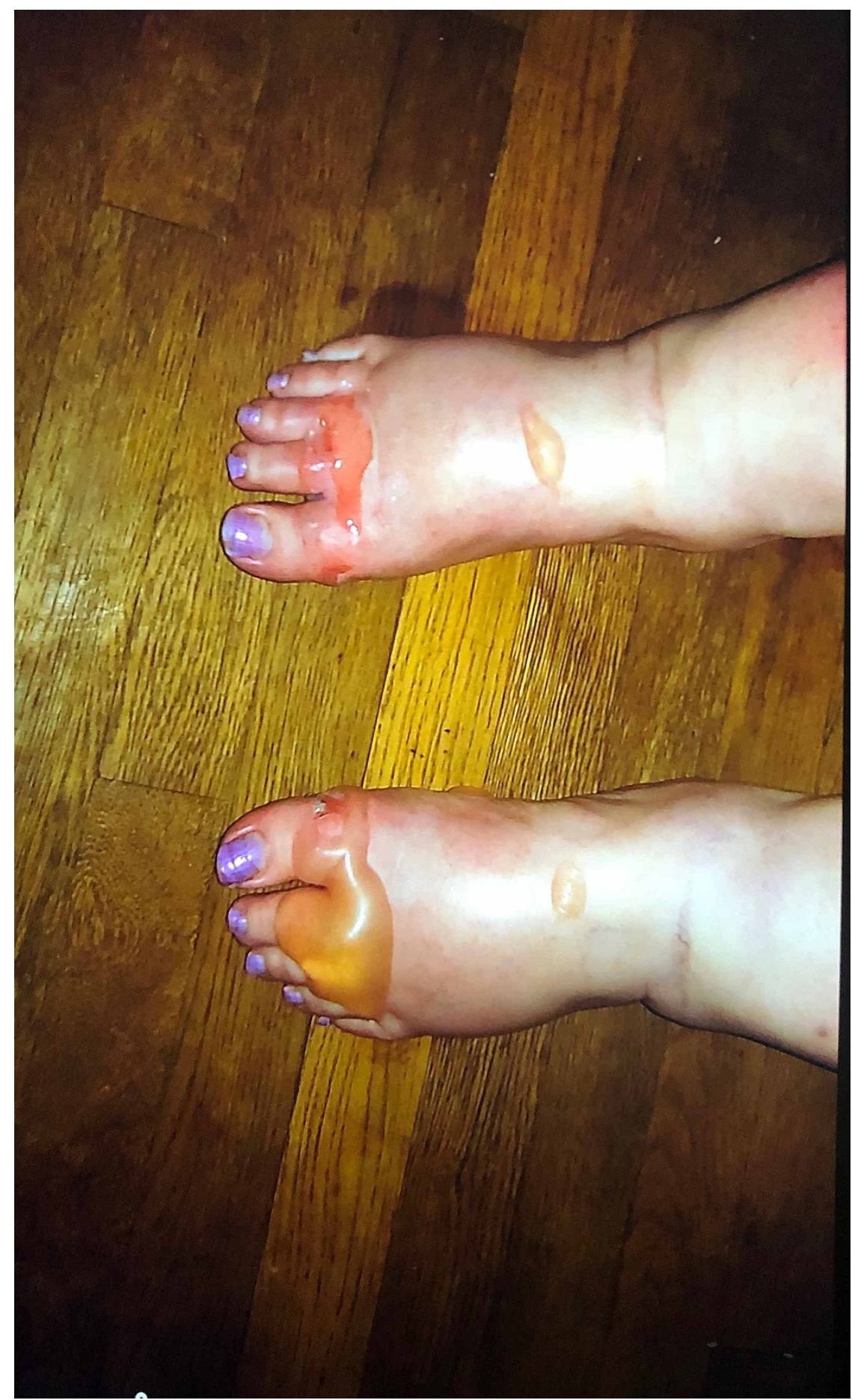

FIGURE 1: Blistering skin lesions on the dorsal surface of both feet. 


\section{Cureus}

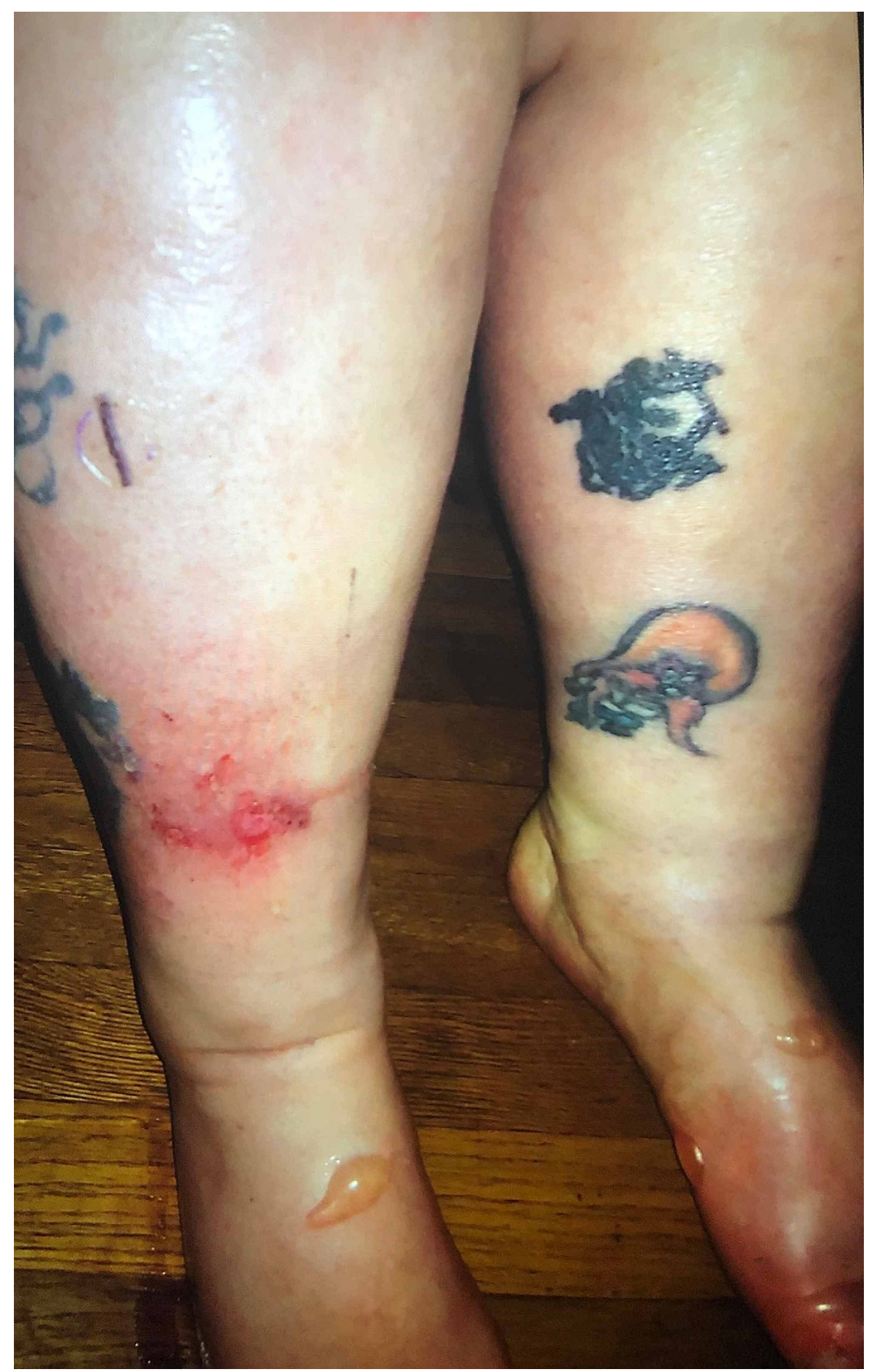

FIGURE 2: Blistering skin lesions on bilateral feet. One ruptured lesion also seen on the lower anterior right shin

According to the patient, the lesions erupted around one week after she started palbociclib. The Nikolsky sign was negative. No evidence of oral mucosal involvement was seen. Palbociclib was immediately discontinued, and she was admitted to the hospital for close monitoring. No immunosuppressive agents, including topical corticosteroids, were administered. The bullous lesions started to erupt in the following days, and no new lesions were seen (Figure 3). She was eventually discharged from the hospital. Since palbociclib was the only medication started around the time of bullous eruptions and improvement occurred after abrupt discontinuation, we strongly believe these skin lesions were associated with it. Biopsy of the lesions could not be obtained due to personal reasons of the patient. 


\section{Cureus}

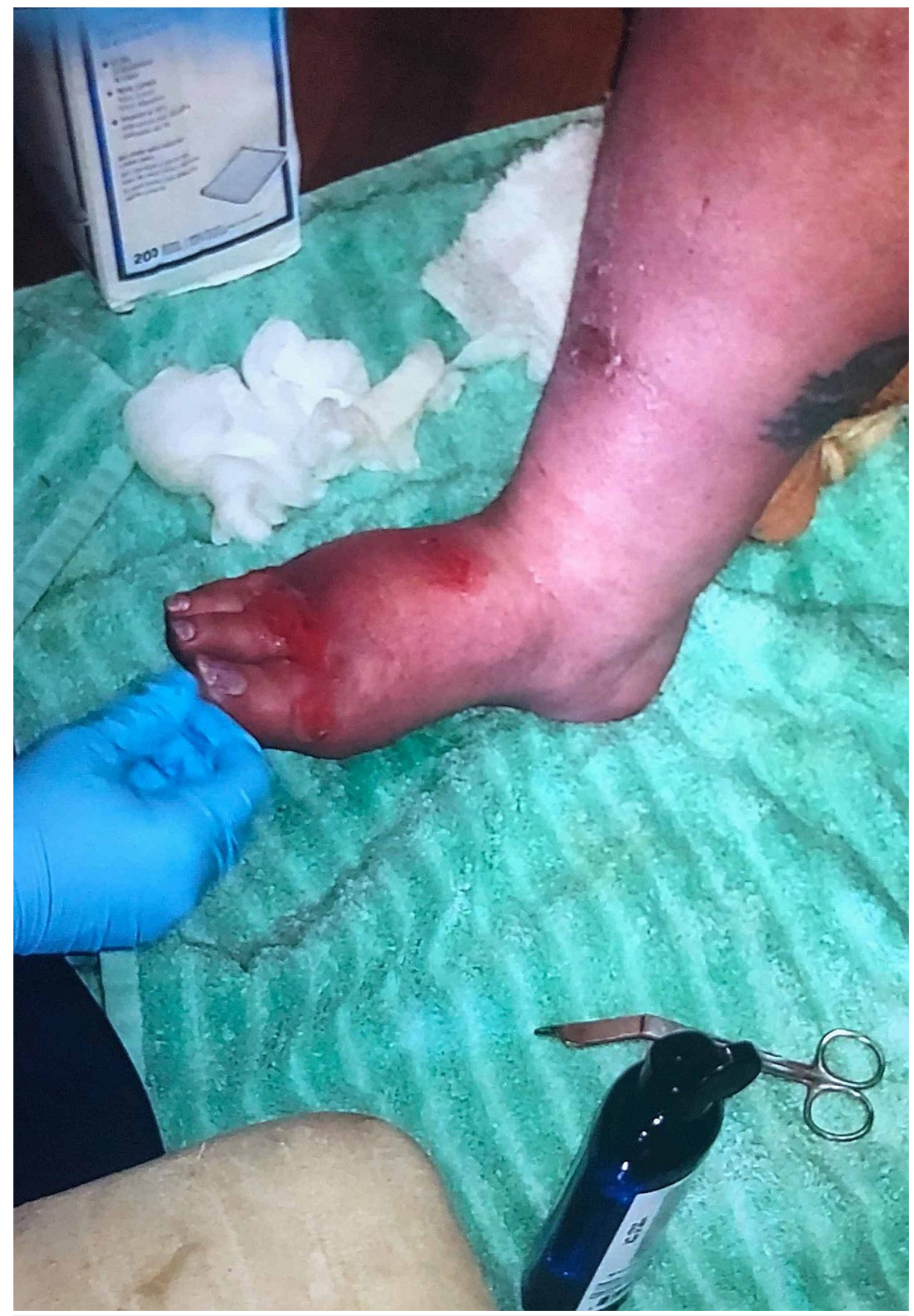

FIGURE 3: Improving blistering skin lesions seen on the right foot two weeks after discontinuation of palbociclib.

\section{Discussion}

Activation of CDK promotes cell cycle progression leading to abnormal cell growth. First-generation CDK inhibitors were initially studied for metastatic hormone receptor (HR+) breast cancer, but due to their panCDK inhibitions, numerous unacceptable toxicities were reported and their use was abandoned [2].

Therefore, selective CDK 4/6 inhibitors, such as palbociclib, abemaciclib, and ribociclib were introduced [3]. All CDK 4/6 inhibitors in combination with aromatase inhibitors are standard-of-care first-line agents in metastatic HR+ breast cancer [4-6].

CDK 4/6 inhibitors are associated with numerous AEs. Cytopenias are one of the most commonly reported AEs with these agents [7]. Palbociclib and ribociclib cause neutropenia in almost $70 \%$ of cases. Abemaciclib is very frequently associated with significant diarrhea [3], whereas ribociclib is known to cause QTC prolongation [8]. Skin reactions have been reported with CDK 4/6 inhibitors but are usually milder and do not warrant discontinuation of the treatment. However, bullous pemphigoid (BP) or bullous skin lesions 
have never been reported with these agents in literature. BP is an immune-mediated skin reaction that has been linked to several drugs, including mefenamic acid [9], etanarcept [10], terbinafine [11], and ampicillin [12]. More recently, dipeptidyl peptidase IV inhibitors (gliptins) have been found to cause BP [13]. Fullblown BP is usually characterized by the presence of tense blistering lesions with desquamative gingivitis involving the mucosal surfaces, but localized lesions are also seen in $10 \%$ to $20 \%$ of cases [14]. Suspicious lesions should be biopsied and evaluated via direct immunofluorescence (DIF), which is the most sensitive test for bullous autoimmune diseases [15].

As mentioned in the case history, a perilesional biopsy could not be obtained in our patient, but classically appearing tense blisters without any other obvious predisposing risk factors except for recent initiation of palbociclib were highly suggestive of drug-induced localized autoimmune bullous disease. Identification of the offending medication is crucial and warrants immediate discontinuation of the agent. Studies have shown that localized bullous disease could be an early manifestation of an advanced and life-threatening BP; therefore, early identification of these lesions is essential [14]. Palbociclib was immediately discontinued in our patient.

High-potency topical corticosteroids are the mainstay of treatment. In one study, high-intensity topical steroid therapy was found to be superior to systemic steroids [16]. In some cases, glucocorticoid-sparing agents, such as azathioprine, methotrexate, or mycophenolate mofetil, could also be used [17]. Considering the subacute nature of disease in our patient, she was closely monitored without requiring any immunosuppressive agents. She showed good clinical improvement.

\section{Conclusions}

Palbociclib is a well-known CDK 4/6 inhibitor used widely in metastatic HR+ breast cancer. It is generally well tolerated, with cytopenias as the most commonly reported AEs. Grade 3 skin toxicities are very rare, but early identification of blistering skin lesions and their prompt discontinuation can prevent life-threatening disease and significant patient comorbidity.

\section{Additional Information \\ Disclosures}

Human subjects: Consent was obtained or waived by all participants in this study. Conflicts of interest: In compliance with the ICMJE uniform disclosure form, all authors declare the following: Payment/services info: All authors have declared that no financial support was received from any organization for the submitted work. Financial relationships: All authors have declared that they have no financial relationships at present or within the previous three years with any organizations that might have an interest in the submitted work. Other relationships: All authors have declared that there are no other relationships or activities that could appear to have influenced the submitted work.

\section{References}

1. Karagounis T, Vallurupalli M, Nathan N, Nazarian R, Vedak P, Spring L, Chen ST: Stevens-Johnson syndrome-like eruption from palbociclib in a patient with metastatic breast cancer. JAAD Case Rep. 2018 , 4:452-454. 10.1016/j.jdcr.2017.12.015

2. Jessen BA, Lee L, Koudriakova T, et al.: Peripheral white blood cell toxicity induced by broad spectrum cyclin-dependent kinase inhibitors. J Appl Toxicol. 2007 , 27:133-142. 10.1002/jat.1177

3. Liu M, Liu H, Chen J: Mechanisms of the CDK4/6 inhibitor palbociclib (PD 0332991) and its future application in cancer treatment. Oncol Rep. 2018, 39:901-911. 10.3892/or.2018.6221

4. Goetz MP, Toi M, Klise S, Frenzel M, Bourayou N, Di Leo A: MONARCH 3: A randomized phase III study of anastrozole or letrozole plus abemaciclib, a CDK4/6 inhibitor, or placebo in first-line treatment of women with HR+, HER2-locoregionally recurrent or metastatic breast cancer (MBC). J Clin Oncol. 2015, 33:TPS624.

5. Finn RS, Martin M, Rugo HS, et al.: Palbociclib and letrozole in advanced breast cancer. N Engl J Med. 2016, 375:1925-1936. 10.1056/NEJMoa1607303

6. Im SA, Lu YS, Bardia A, et al.: Overall survival with ribociclib plus endocrine therapy in breast cancer . N Engl J Med. 2019, 381:307-316. 10.1056/NEJMoa1903765

7. Anders CK, LeBoeuf NR, Bashoura L, Faiz SA, Shariff AI, Thomas A: What's the price? toxicities of targeted therapies in breast cancer care. Am Soc Clin Oncol Educ Book. 2020, 40:55-70. 10.1200/EDBK 279465

8. Santoni M, Occhipinti G, Romagnoli E, et al.: Different cardiotoxicity of palbociclib and ribociclib in breast cancer: gene expression and pharmacological data analyses, biological basis, and therapeutic implications. BioDrugs. 2019, 33:613-620. 10.1007/s40259-019-00382-1

9. Shepherd AN, Ferguson J, Bewick M, Bouchier IA: Mefenamic acid-induced bullous pemphigoid. Postgrad Med J. 1986, 62:67-68. 10.1136/pgmj.62.723.67

10. Kluk J, Goulding JM, Bhat J, Finch TM: Drug-induced bullous pemphigoid: cases triggered by intravenous iodine and etanercept. Clin Exp Dermatol. 2011 , 36:871-873. 10.1111/j.1365-2230.2011.04102.x

11. Aksakal BA, Özsoy E, Arnavut Ö, Gürer MA: Oral terbinafine-induced bullous pemphigoid. Ann Pharmacother. 2003 , 37:1625-1627. 10.1345/aph.1D013

12. Hodak E, Ben-Shetrit A, Ingber A, Sandbank M: Bullous pemphigoid-an adverse effect of ampicillin. Clin Exp Dermatol. 1990, 15:50-52. 10.1111/j.1365-2230.1990.tb02020.x

13. Skandalis K, Spirova M, Gaitanis G, Tsartsarakis A, Bassukas ID: Drug-induced bullous pemphigoid in diabetes mellitus patients receiving dipeptidyl peptidase-IV inhibitors plus metformin. J Eur Acad Dermatol 


\section{Cureus}

Venereol. 2012, 26:249-253. 10.1111/j.1468-3083.2011.04062.x

14. Tran JT, Mutasim DF: Localized bullous pemphigoid: a commonly delayed diagnosis . Int J Dermatol. 2005, 44:942-945. 10.1111/j.1365-4632.2004.02288.x

15. Pohla-Gubo G, Hintner H: Direct and indirect immunofluorescence for the diagnosis of bullous autoimmune diseases. Dermatol Clin. 2011, 29:365-372. 10.1016/j.det.2011.03.001

16. Joly P, Roujeau JC, Benichou J, et al.: A comparison of oral and topical corticosteroids in patients with bullous pemphigoid. N Engl J Med. 2002, 346:321-327. 10.1056/NEJMoa011592

17. Thai KE: Fitzpatrick's dermatology in general medicine, 7th edition. Aust J Dermatol. 2008, 49:175. 10.1111/j.1440-0960.2008.00459.x 